\title{
A free and simple computerized screening test for visual field defects
}

Nordfang, Maria; Uhre, Valdemar; Robotham, Ro Julia; Kerry, Sheila J.; Frederiksen, Jette Lautrup; Starrfelt, Randi

Published in:

Scandinavian Journal of Psychology

DOI:

10.1111/sjop. 12546

Publication date:

2019

Document version

Peer reviewed version

Citation for published version (APA):

Nordfang, M., Uhre, V., Robotham, R. J., Kerry, S. J., Frederiksen, J. L., \& Starrfelt, R. (2019). A free and simple computerized screening test for visual field defects. Scandinavian Journal of Psychology, 60(4), 289-294.

https://doi.org/10.1111/sjop.12546 


\title{
A free and simple computerized screening test for visual field defects
}

\author{
Maria Nordfang ${ }^{a *}$, Valdemar Uhre ${ }^{\text {b,c,d }}$, Ro Julia Robotham ${ }^{\mathrm{e}}$, Sheila Kerry ${ }^{\mathrm{f}}$, \\ Jette Lautrup Frederiksen ${ }^{\mathrm{a}, \mathrm{d}}$ and Randi Starrfelt ${ }^{\mathrm{e}}$ \\ ${ }^{a}$ Department of Neurology, Rigshospitalet, Glostrup, Denmark; ${ }^{b}$ Danish Research \\ Center for Magnetic Resonance, Hvidovre, Denmark; ' Research Unit, Child and \\ Adolescent Mental Health Centre, Copenhagen, Denmark; ${ }^{d}$ Department of Clinical \\ Medicine, Faculty of health Sciences, University of Copenhagen, Denmark. \\ ${ }^{e}$ Department of Psychology, University of Copenhagen, Denmark; Institute of Cognitive \\ Neuroscience, University College London, UK \\ maria.nordfang@regionh.dk
}

Paper in press in Scandinavian Journal of Psychology [2019]. This paper is not the copy of record and may not exactly replicate the authoritative document published.

DOI: $10.1111 /$ sjop. 12546 


\section{A free and simple computerized screening test for visual field defects}

- Introduction: About 30-40 \% of stroke patients suffer from visual field defects following injury. These can interfere with the standard neuropsychological assessment and complicate the interpretation of tests that use visual materials. However, information about the integrity of a patient's central visual field is often unavailable. We therefore designed a screening tool, the computerized visual field test (c-VFT), specifically targeted at providing easily available, but rough, information about patients' central visual field.

- Method: c-VFT was tested in two samples of stroke patients. 11 patients were tested on c-VFT and on the Esterman test. 5 patients were tested on c-VFT and the Humphrey Visual Field Analyzer (HFA), central 10-2. Criterion validity of the c-VFT was investigated by calculating quadrantwise intraclass correlation for both comparisons. For the HFA comparison, we also calculated point-to-point intraclass correlation, sensitivity, and specificity.

- Results: Analyses revealed moderately good correspondence between c-VFT and the Esterman test, and between c-VFT and HFA 10-2 respectively. When looking specifically at test points within one degree of visual angle apart in the two tests, intraclass correlation increased. For these points, the sensitivity of c-VFT was .89 and specificity was .97 .

- Conclusions: While the c-VFT is not designed to be diagnostic nor to replace the detailed visual field analysis, this study shows that it provides a reasonable screening of the central visual field. The test can easily be used and will be made freely available to neuropsychological clinicians and researchers.

Keywords: visual field screening, hemianopia, stroke, anopia, neuropsychological assessment 


\section{Introduction}

Visual field defects are common after stroke (Neumann et al., 2016; Zihl, 2011). As visual materials are often used for neuropsychological assessment of stroke patients, it is important that the neuropsychologist be aware of visual field defects that may affect the patient's performance on tests using visually presented information (e.g., Rowe et al., 2009). Based on knowledge of the patient's current visual field, the neuropsychologist can adapt the presentation of visual material and chose the tests best suited for the particular patient. However, such information is not always available. While confrontation visual field examination is a standard part of the neurological examination, it is not very sensitive for small or shallow defects (Panditt, Gales, \& Griffiths, 2001). In addition, often only one location in each quadrant is tested in foveal vision. This is problematic, as even small, central visual field defects are very important for reading and analyzing visual information presented on paper or computer, such as neuropsychological test materials. It has also been shown that visual field defects can have a devastating effect on the patients' ability to return to the work and on quality of life (e.g., Ali et al., 2013; Hepworth \& Rowe, 2016).

There are various challenges related to acquiring sensitive visual field assessments during hospitalization. Logistic issues, such as waiting times, can have the consequence that an ophthalmological examination is either not performed at all or that it is scheduled after the neuropsychological assessment. Also, many neurological patients are not able to cooperate to full automated perimetry as these tests are often cognitively straining and require that the patient is able to sit still in a regular chair. Visual field defects following stroke can change substantially over the first weeks and months (see Zihl, 2011 for examples). Thus, even when a thorough visual field analysis is available for the patient this may not always represent the current function of the 
patient's visual field. All of the above can have the consequence that information about the patient's visual field is lacking at the time of the neuropsychological examination. In these situations, a screening test that is easy to use for patients as well as neuropsychologists may prove valuable.

A number of computerized visual field screening tests already exist (e.g., Dzwiniel et al., 2017; Koiava et al., 2012; Olsen et al., 2016). However, most of these run online (e.g., Koiava et al., 2012 and Olsen et al., 2016) and thus require a stable internet connection to work as intended. Other programs, while giving excellent information on the patient's visual field, are harder to complete for the patients or harder to administer for the examiner (e.g., Dzwiniel et al., 2017). Test selection and the interpretation of results often require in-depth knowledge about visual perception, detailed knowledge about visual field assessment techniques, as well as advanced computer skills. Consequently, the tests are not always well-adapted for a general neuropsychologist to use.

To our knowledge no validated test has been developed with the specific aim of screening patients prior to neuropsychological assessment. We have therefore designed a short screening test (the "computerized visual field test", c-VFT) for the central visual field that is easy to complete for patients, easy to administer, runs offline in freely available software, and presents results in a manner that are readily available and easy to interpret. The test is brief - it takes approximately 5,5 minutes for patients to complete it under the standard settings. Furthermore, the spatial layout of the test points is specifically designed to target areas that are important for reading and recognizing materials presented on a sheet of paper or on a computer screen. As the test targets the central 20 degrees of visual angle with a high resolution foveally, it can be seen as an 
excellent addition to the manual perimetry that is often performed as a standard with neurological patients.

The c-VFT is intended to give neuropsychologists a rough idea of a patient's visual field prior to neuropsychological assessment. The test is not designed as a diagnostic tool and is therefore not appropriate to use for diagnostic purposes.

In order to validate the c-VFT, we compared it against gold-standard visual field tests in two settings.

\section{Computerized Visual Field Test (c-VFT)}

The computerized visual field test is programmed in PsychoPy ver 1.90.2 (Peirce, 2007, 2009) and tested on Windows. PsychoPy is an open source application written in Python. It is freely available to download and install, it runs offline, and is platform free and runs on both iOS, Linux and Windows.

The c-VFT can be downloaded by professionals from https://www.psy.ku.dk/ansatte/?pure=da\%2Fpublications\%2Fcvft(c39487b2-2ad14cb0-bf6d-73d79eb87a06).html. The test probes whether participants can detect briefly presented dots within the central 20 degrees of the visual field.

The default settings of the test are described below. In addition to the default settings, a number of test parameters can be changed by the investigator to customize the test to the individual participant and the test situation. These advanced options are described after the description of the default procedure.

\section{Stimuli.}

The test probes 48 points within a radius of 10 degrees of visual angle (dva) around a central fixation cross. The points are equally-sized dark circles (RGB: 64, 64, 64; luminance: $0.12 \mathrm{~cd} / \mathrm{m}^{2}$ ) presented against a light-grey background (RGB: 192, 192, 192 , luminance: $143 \mathrm{~cd} / \mathrm{m}^{2}$ ). Discounting ambient light, this results in a Weber contrast of - 
0.999. Each point extends $2 \times 2$ millimeter. A fixation cross is placed in the center of the screen. The cross is $1 \mathrm{dva}$, red (RGB: $255,0,0$, luminance: $48 \mathrm{~cd} / \mathrm{m}^{2}$ ) and occasionally changes color to green (RGB: $0,255,0$; luminance: $187 \mathrm{~cd} / \mathrm{m}^{2}$ ). The reported luminance values are based on the Danish setup. These values will vary dependent on the specific set up; the specific screen, graphics card and ambient lighting. The c-VFT is designed to be a fast and easy screening tool; consequently, luminance control is not a feature of the test. Rather, the screening test is intended to be useful under lax testing conditions.

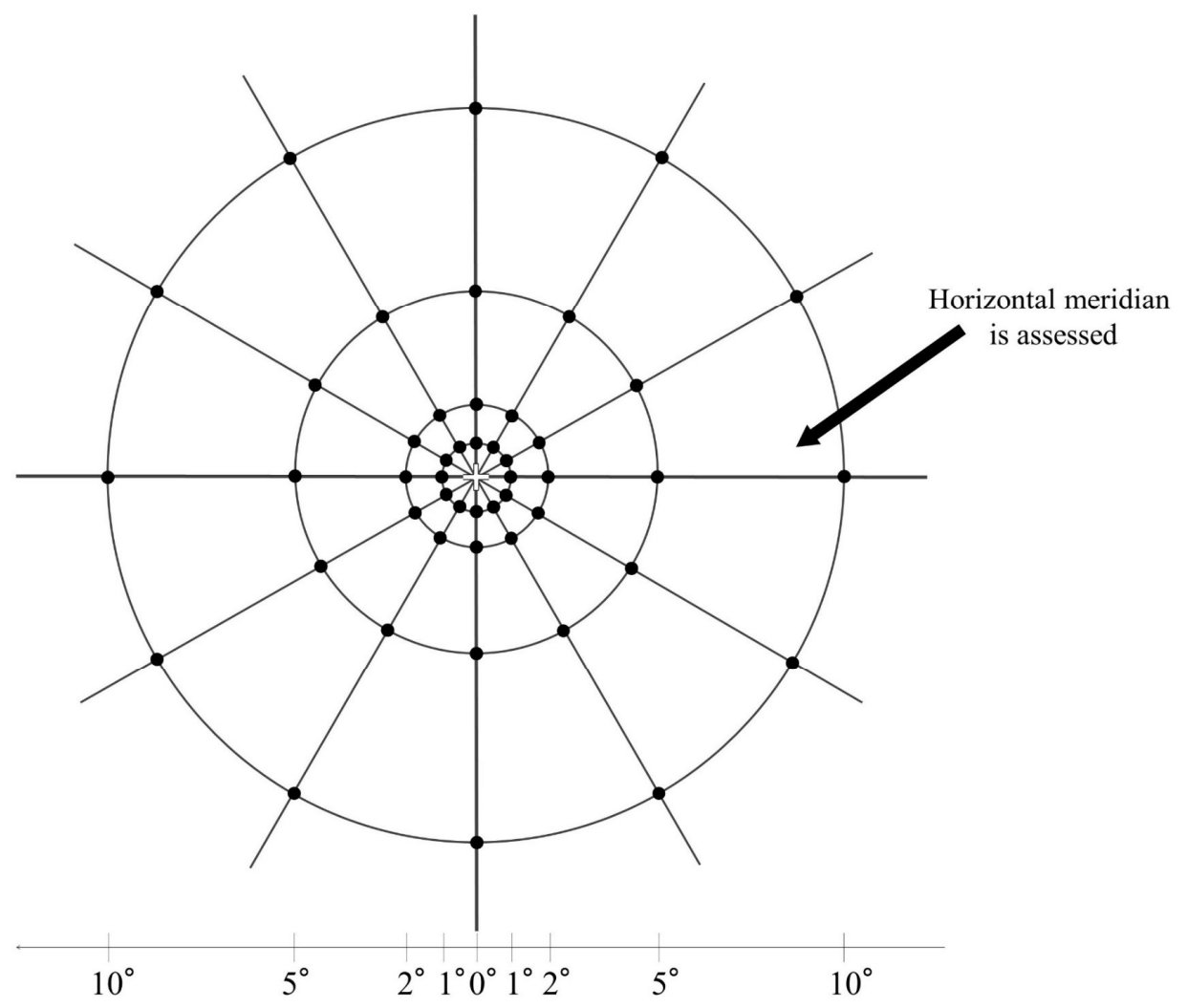

Figure 1. Illustration of the spatial setup of the c-VFT. Black dots indicate locations that are probed during testing. The concentric circles are drawn for illustration and are not visible during testing. Colors differ from the actual test. 
The 48 test points are placed on the circumferences of four imaginary concentric circles (12 equidistantly spaced points on each circle). The radiuses of the four circles span 1 , 2,5 , and $10 \mathrm{dva}$. The points are placed such that the horizontal and vertical meridians are tested. The horizontal meridian is particularly important for reading; consequently, the test is expected to be sensitive for visual field defects that affect reading abilities (see figure 1). This setup may, however, be changed in the advanced settings (see below).

\section{Procedure.}

Participants are seated in front of the monitor at a viewing distance of $60 \mathrm{~cm}$. Participants are instructed to fixate on a centrally presented cross throughout the test, and they are asked to respond as quickly as possible whenever they see a dot appearing. To ensure central fixation, a response is also required when the fixation cross changes color from red to green. A color change is particularly hard to detect if not fixated as color vision rapidly decrease with eccentricity. Responses are made by pressing the space bar on the keyboard. Responses made within 2 seconds after presentation of a dot are counted as correct. Presentation of each dot is terminated after 2 seconds, or when (and if) a response is made. The inter stimulus interval (ISI) varies randomly between 1, 2 , and 3 seconds, with the constraint that each duration is used an equal amount of times. Consequently, if a participant randomly presses the spacebar, half of the responses will on average be correct and half will be false alarms. A change in fixation color happens six times for each run of 48 test points. In the default settings, participants go through two repetitions; i.e., each test point is probed twice, and the total number of trials is 108 . Test points and fixation points are presented in random order. 
Before the test starts, the investigator can provide subject name, age, handedness and set the language of the test (English or Danish). The investigator also determines whether the test should start with a practice session, and whether he/she wishes to use advanced settings. The first time the test is run on a particular computer, the test starts with a short screen fitting procedure. Here, a standard credit card is matched to a frame on the screen. The investigator places the card on the screen and adjusts the size of the frame by pressing the arrow keys. This procedure ensures that it is very easy to calibrate any screen for correct presentation of the test. The screen size must be at least $22 \times 22$ $\mathrm{cm}$. The test then starts with an instruction screen and is by default followed by a short practice phase in which four points (one in each quadrant) and one fixation change is presented. The investigator can choose to repeat the practice phase if this is deemed

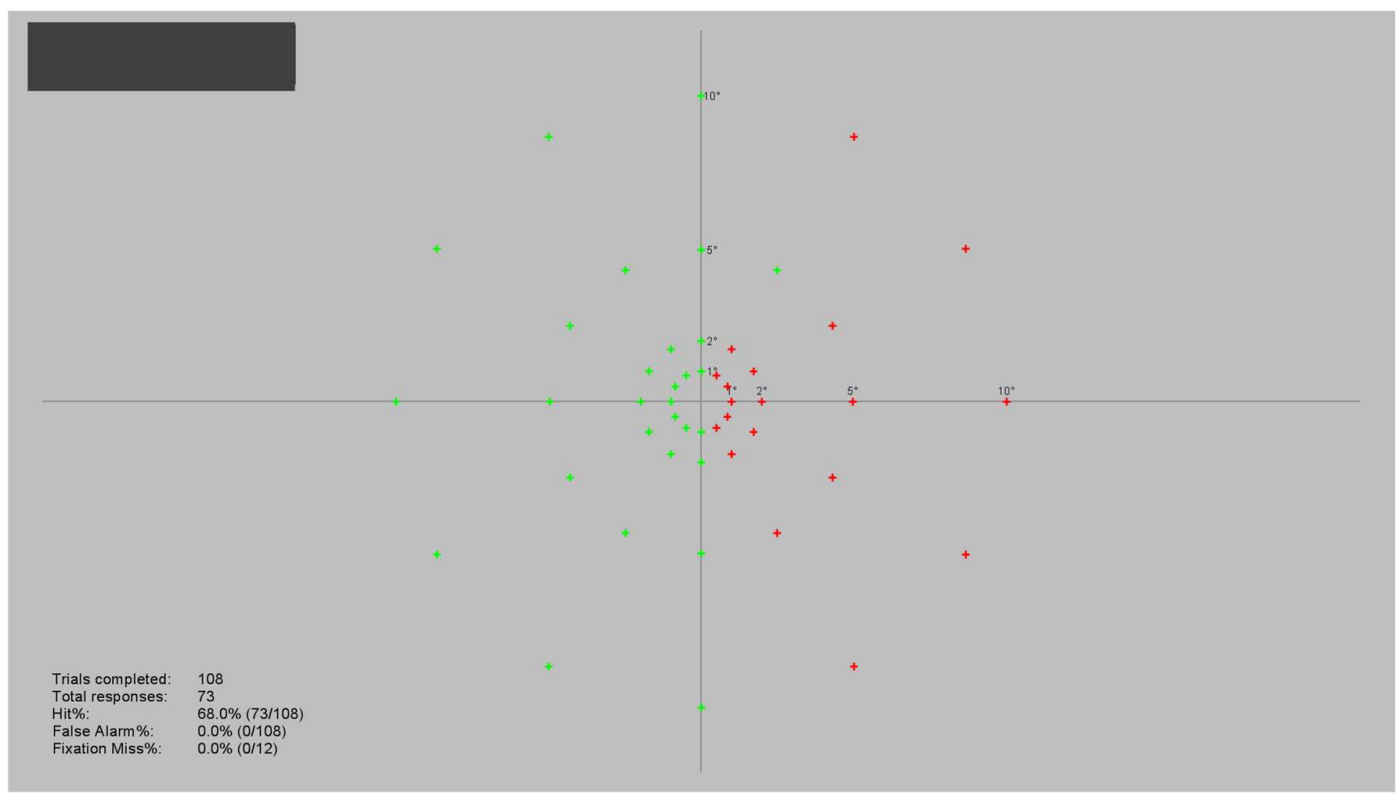

necessary for correct completion of the test. The test ends with a results screen providing a visual illustration of the results where the number of correct responses for each of the probed locations is color coded, and basic information on the responses during the test are noted (see Figure 2). The results screen is saved as a .pdf-file, and all data from the test is saved in a .dat-file for optional further analysis. 
Figure 2. Results screen c-VFT. The responses for each location are color coded for immediate interpretation. An overview of the response data is given in the bottom left corner. Patient ID and time and date are noted in the top left corner (here masked for anonymity).

\section{Advanced settings.}

While the standard settings are sufficient for a screening of the visual field, some advanced settings are also available for more detailed and flexible testing. Several test parameters can thus be changed in the advanced settings option:

- The color of the fixation cross can be changed to blue, to accommodate for redgreen color blindness.

- The display can be rotated so that the meridians are not tested.

- The size of the test points can be set at 1,2, 3, 4, or 5 millimeters.

- The test can be run in a high-contrast version with white (RGB: 255, 255, 255) dots presented against a black (RGB: $0,0,0)$ background.

- The number of probes at each test location can be set at 2, 3, or 4 .

- The length of the valid response window can be set at 2, 3, or 4 seconds.

- $\quad$ The exposure duration of test points can be set to values between 0.1 and 2.0 seconds in increments of 0.1 .

Some of these advanced parameter changes will make the test easier, such as increasing the size and contrast of the stimuli and increasing the response time window. These settings provide a mean to investigate a patient population who may not always be able to comply with the default settings. Note that using the high contrast version of the test is specifically suitable for investigating deep visual field defects but will be less valid 
for patients with more shallow visual field defects where some information may still be processed.

Other of the advanced parameters target specific conditions that the experienced neuropsychologist or researcher may be interested in. None of the advanced settings are validated here and should be used and interpreted with caution.

\section{c-VFT and Esterman test}

Performance on the c-VFT was compared against results from the Esterman test (Esterman, 1982), which is widely used internationally for assessing visual field defects in relation to driving capabilities. Stroke patients with visual field defects who were scheduled to take the Esterman test at the Aphaisa Lab at University College London were given the option to participate in the present study.

\section{Materials and method}

Participants. Prospective participants were identified at the specialist Hemianopia clinic at the National Hospital for Neurology and Neurosurgery, University College London. The testing took place as part of participation a larger study granted ethics by the East Midlands - Leicester Central Research Ethics Committee.

Eleven patients (eight men) with suspected visual field defects agreed to participate and provided informed written consent. Mean age was 64 years, and mean time since stroke was 9 months for the completion of the Esterman test, and 15 months for the completion of the c-VFT. Consequently, patients were in the chronic phase and spontaneous remission was expected to be minimal.

Apparatus and setup. The binocular Esterman test (cf. Esterman 1982) was carried out on an Octopus 900 perimeter. The c-VFT was run binocularly, on a standard desktop computer (BenQ XL2430T: 24 inch, using resolution $1920 \times 1080)$ in a room with 
standard ceiling light. Participants first completed the mandatory Esterman test and took part in the c-VFT at a subsequent visit. Due to the recruitment procedure, order of testing was not random. This is considered in the discussion.

Analysis. A common challenge when validating two visual field tests against each other is that they rarely probe the exact same locations in space (see e.g., Dzwiniel et al., 2017 and Koiava et al, 2015 for other studies that have faced the same challenge). The Esterman test covers an oval space, vertically spanning 30 degrees of visual angle superior, 60 degrees inferior and horizontally spanning 160 degrees. It probes 120 points within this space, however only ten points lie within the central 20 degrees. The c-VFT tests a circular space with a diameter of 20 degrees (cf. Figure 1). Consequently, only some of the test points in the two tests concern the same section of the visual field. In the analysis, we focus on the test points that lie within the same perimeter on both tests (cf. Figure 3). For the Esterman test, we use the detection scores for the ten points that overlap with the test area of the c-VFT. We test the c-VFT's criterion validity by calculating intraclass correlation (ICC) for each quadrant, thereby accommodating the fact that 10 points in the Esterman test corresponds to 48 points in the c-VFT. Following Koo and Li (2016) and Shrout and Fleiss (1979) our ICC model is a two-way mixed effects, single measurement model with two raters, i.e., ICC $(3,1)$. Any single missed probe in any of the locations in a quadrant means that this quadrant is classified as damaged. Meridians are not included in the classification, as they border two quadrants and do not clearly pertain to one or the other. By this analysis we investigate whether the Esterman and c-VFT agrees whether a quadrant is damaged or not. 


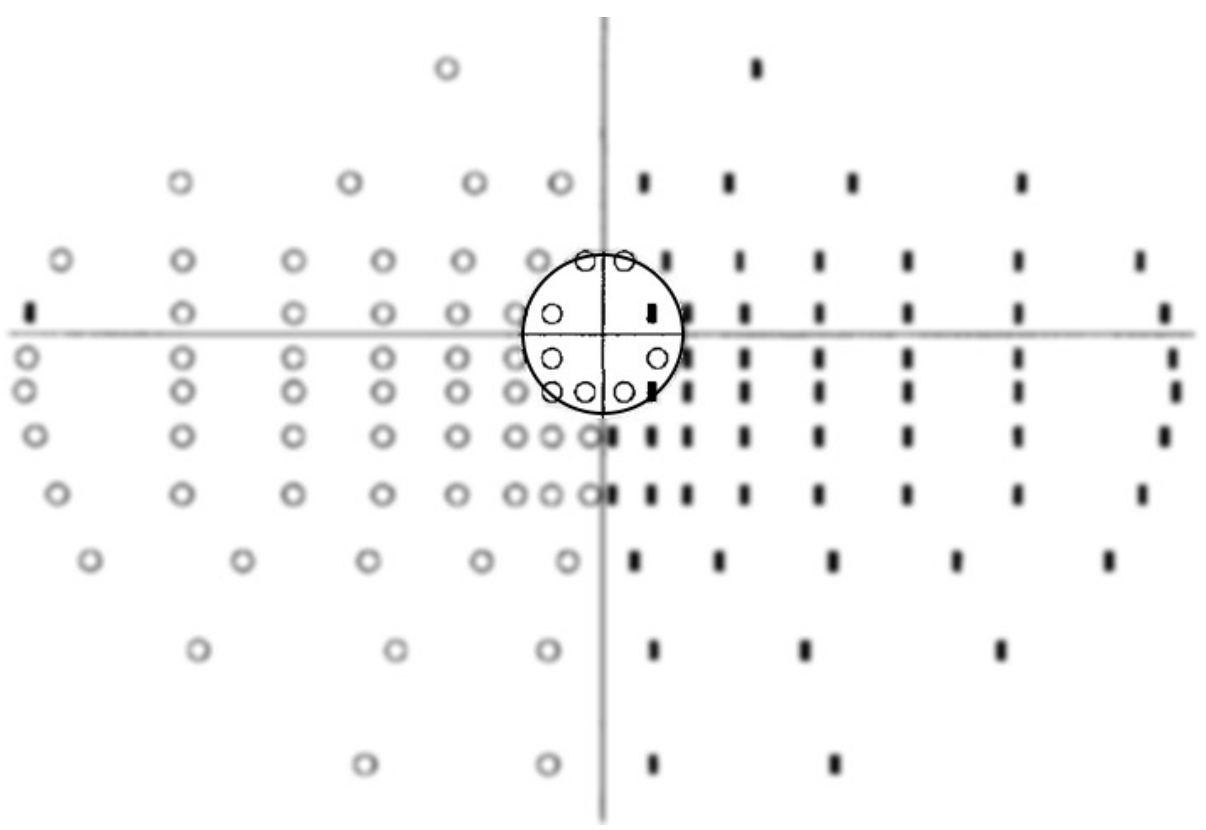

Figure 3. Example of the output from the Esterman test in comparison with the c-VFT. The circles indicate locations that were responded to when probed. The rectangular shapes indicate missed locations. The large circle encompasses the locations that are within $10 \mathrm{dva}$ from center and thus the overlap between the Esterman test and the cVFT.

\section{Results and discussion}

ICC between the c-VFT and the Esterman test revealed a moderately good correlation $(\mathrm{ICC}=.53)$ between the two tests. The correlation was highly significant $p<.001, \mathrm{CI}=$ .28 - .72. Inspection of the contingency table (Table 1 ) revealed that in all cases where the Esterman test showed a deficient response the c-VFT also measured a deficit. The Esterman test was always carried out prior to the c-VFT. Thus, it appears that there was no re-test effects nor evidence of spontaneous remission between the two consecutive tests. Conversely, in several cases the c-VFT measured defects that were not picked up by the Esterman test. This is not surprising as the number of locations within the 10 degree perimeter that are tested in the Esterman test is considerably smaller than in the 
c-VFT (i.e., ten versus 48 points). It is likely that the c-VFT measured real visual field defects in the central visual field that are not probed in the Esterman test, at least in some patients. 8 patients showed a deficit on the c-VFT that was not picked up by the Esterman tests. Two of these patients also missed a few (two or three) fixations probes, and for these specific patients, eye movements may have contributed to their missing some of the central test points.

Table 1. Contingency table for the Esterman and c-VFT classification of quadrantwise defects. A zero indicates no defect, a one indicates a defect.

\begin{tabular}{lccc}
\cline { 2 - 3 } & & \multicolumn{3}{c}{ Esterman } \\
& & 0 & 1 \\
\hline $\mathrm{c}-V F T$ & 0 & 22 & 0 \\
& 1 & 12 & 10 \\
\hline
\end{tabular}

\section{c-VFT and Humphrey Automated Visual Field Analyzer}

The comparison between the c-VFT and the Esterman test revealed a promising correlation between the quadrantwise results of the two tests. However, considerable differences between the numbers of centrally located points in the two tests posed a challenge for direct comparison. To replicate and further investigate the criterion validity of our test, we conducted a second comparison. We used the 'gold standard' for visual field testing, namely the Humphrey 750i Automated Visual Field Analyzer (HFA; Carl Zeiss Meditec Inc., 2003) and chose a test with a high resolution in the central visual field - the 'central 10-2' for which all test points lie within a radius of 10 
dva, similar to the c-VFT. We had access to a HFA for patients with visual field defects following stroke at Rigshospitalet, Glostrup in Denmark.

\section{Materials and method}

Participants. 5 patients (all men) with visual field defects following stroke agreed to participate in the study. They were on average 53 years old, and time since injury was in the range from 14 days to five years. Data collection was approved by the data protection committee at Region Hovedstaden, approval number RH-2017-57, I-Suite: 05299, and evaluated by the regional ethics committee, request number 16047073 . All participants gave informed written consent.

Apparatus and setup. The HFA central 10-2 test was run in a dimly lit room, one eye at a time. The c-VFT was run on a stationary computer using an Elo 2201L touch screen, in a room with ambient indoor lightning and transparent curtains drawn. Each point in the c-VFT was probed four times. For all other settings the default was used.

The order of the two tests was randomly drawn for the first participant, and then alternated for the following participants. Three participants started with the HFA test and two with the c-VFT. Both tests were run on the same day.

Analysis. Similar to the comparison with the Esterman test, we calculated the quadrantwise intraclass correlation. For output from the HFA, we used the pattern deviation maps. Here, a score below $1 \%$ in a quadrant for both of the two eyes meant that it was counted as deficient, as the c-VFT is binocular. For output from the c-VFT we again applied the criterion that if any single probe was missed on any location in a quadrant, the quadrant was classified as defect. The HFA deviation plots, are based on threshold testing of missed locations. By this scheme, probe intensity is increased or decreased according to a detailed algorithm and probe locations are repeated based on participants' previous responses at a given location (see Carl Zeiss Meditec Inc, 2003 
for further information). Furthermore, the HFA entails detailed fixation control. The cVFT presents a more static testing regime in which number of repetitions and probe luminance are constant. Consequently, the c-VFT measure is expected to be more suspect to random variations based on for example brief lapses of attention compared to the HFA. The relatively long exposure durations in the c-VFT counteract this vulnerability, and the central fixation task provides a rough measure for task compliance. We return to these points in the results and discussion section.

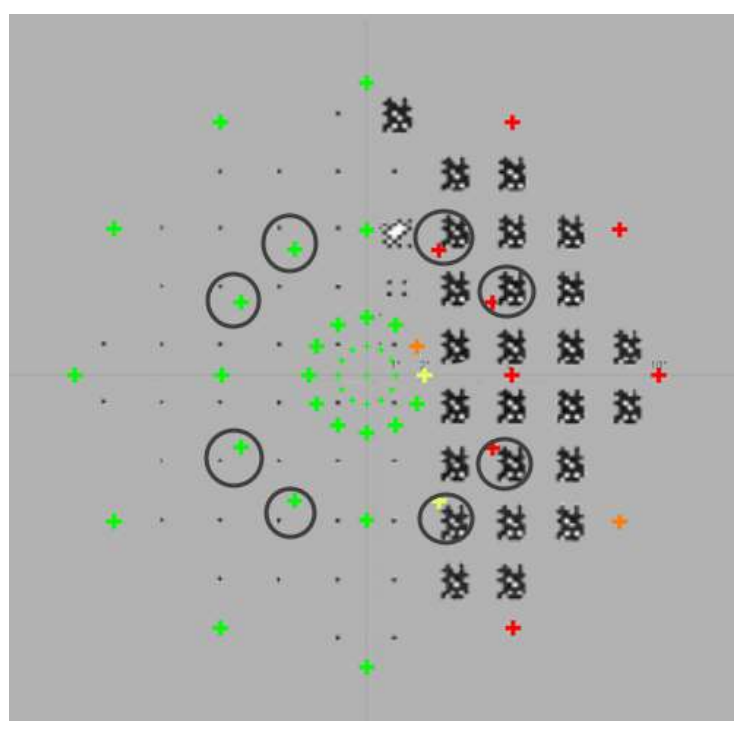

Figure 4. Example of the output from the HFA 10-2 pattern deviation map from one eye (left) from one participant. The HFA output is overlain an illustration of the cVFT output for comparison (the crosses for perimeter 2, 5, and 10 in the illustration are enlarged for easy inspection). The locations that are encircled in the Figure are those locations for which one test point in one test is less than one dva apart from exactly one test point in the other test. These points represent the data used for the pointwise comparison.

In addition to the above analysis, we did a pointwise comparison for the eight test points that were less than 1 dva apart in the two tests, when a single point in the c- 
VFT corresponded to a single point in the HFA (see Figure 4). For the pointwise comparison we also calculated sensitivity and specificity.

\section{Results and discussion}

All participants responded correctly to all the 24 central fixation color changes that were presented during the test. This indicates good test compliance for the participants, and we do not suspect major lapses of attention has occurred during testing. As expected, the quadrantwise intraclass correlation between the c-VFT and the HFA test was better $(\mathrm{ICC}=.65)$ than between the $\mathrm{c}-\mathrm{VFT}$ and the Esterman test. Again, the correlation was highly significant $p<.001, \mathrm{CI}=.31-.85$. The contingency table (Table 2$)$ shows that when the c-VFT revealed a defect in a quadrant, then this defect was confirmed in the HFA test. However, occasionally (four times) the HFA test detected defects that were not picked up by the c-VFT. As opposed to the Esterman test, the HFA tests a more finely dispersed grid than the c-VFT in most parts of the central visual field.

Consequently, the HFA may be picking up defects in areas that are not probed by the cVFT. Also, the HFA is thoroughly researched to have excellent detection rates and it applies a monocular testing procedure, all of which may contribute to greater sensitivity. No defects were detected only in the c-VFT. Although the c-VFT is suspected to be more vulnerable to random variations in responses, we see no indication of this in the data. 
Table 2. Contingency table for the HFA 10-2 and c-VFT classification of quadrantwise defects. A zero indicates no defect, a one indicates a defect.

HFA $10-2$

\begin{tabular}{llcc} 
& 0 & 1 \\
\hline $\mathrm{c}-\mathrm{VFT}$ & 0 & 6 & 4 \\
& 1 & 0 & 10 \\
\hline
\end{tabular}

Remarkably; when we look specifically at the test points that correspond more directly to each other in the two tests (i.e., a point in one test lie within less than $1 \mathrm{dva}$ from exactly one point in the other test), the intraclass correlation between the two tests increase to $\mathrm{ICC}=.74, p<.001, \mathrm{CI}=.56-.85$. An intraclass correlation of $>.75$ indicates excellent correlation.

The point-to-point contingency table (Table 3) reveal that, overall, three points that were classified as defect in the HFA were not registered by the c-VFT, whereas only one point was classified as defect in the c-VFT and not in the HFA. Taking the HFA as the gold standard we calculated the sensitivity of the c-VFT to be .89 , and the specificity was .97 . This suggests that the c-VFT does not detect all defect locations. However, when a probe is missed in the c-VFT this indeed indicates an underlying defect and should warn the neuropsychologist that the patient's visual field may not be intact in this area. 
Table 3. Contingency table for the HFA 10-2 and c-VFT classification of pointwise defects. A zero indicates no defect, a one indicates a defect.

\section{HFA $10-2$}

\begin{tabular}{lccc} 
& & 0 & 1 \\
\hline c-VFT & 0 & 8 & 1 \\
& 1 & 3 & 28 \\
\hline
\end{tabular}

\section{Discussion}

The aim of this study was to investigate the validity of a new visual field screening tool specifically targeted for use in the neuropsychological clinic. The c-VFT is a brief screening tool that only takes approximately 5,5 minutes to complete using the standard settings. It is easy to administer as it does not require an internet connection, has a practical screen calibration tool and standard settings. It also includes a simple output screen visually illustrating the results, that makes it is easy to interpret performance on the test immediately after the test has been completed and communicate the results to the patient. The test does not require participants to sit in a regular chair, making it wellsuited for patients with mobility limitations. The idea behind the test is to provide a screening tool that can be used by neuropsychologists off line and on the fly to compliment neuropsychological testing. The test is not suitable for diagnosis. It is brief and designed to give a rough idea of the patients' visual field. We make no attempt of controlling luminance of the stimuli. When the c-VFT is run on different makes of computers using different graphics cards and screens, the exact presentation of the stimuli will vary. We have validated the test in different settings, using a lenient design without luminance testing, head fixation, lighting control, and eye tracking. Even under 
these noisy testing conditions the c-VFT compares well to highly controlled, 'gold standard', automated perimetries. The data thus suggest that the test provides a fairly robust tool for quickly screening for visual field defects. However, it is important to note, that the short screening tool cannot replace detailed visual field analysis. The cVFT is not a diagnostic tool. It is designed solely to aid neuropsychological assessment. A limitation of the current study is that patient recruitment was based on willingness to participate. Consequently, it is likely that the level of functioning of the included patients is relatively high. The reported sensitivity and specificity of c-VFT may potentially be higher for the relatively well functioning sample in the present study than for the broader population of patients with recently acquired visual field defects following brain injury. Participants with encompassing cognitive deficits may present more noisy data and reduced compliance for both automated perimetries and the c-VFT. In the c-VFT, these difficulties will often be evident from the patients' ability to respond to the central fixation task. Thus, we recommend discarding the test if there are errors in the central fixation task. A caveat of this procedure is that patients with central visual field loss may potentially impair the performance on the central task, as only half of the cross will be visible. We see no indication of this problem in the present data, as all participants responded perfectly to the central fixation task. Nevertheless, based on the risk of misinterpreting the results when the central fixation task has not been responded to, we recommend discarding the test in this case. Even though this theoretically can mean discarding a useful test occasionally.

Overall, the c-VFT test shows good correlation with standard visual field analyzers across two different comparisons, even as the area of coverage varies between the tests. When analyzing results from the visual areas with direct correspondence between two tests (for the HFA and c-VFT) we find very good correlation, good 
sensitivity and excellent specificity. Thus, when patients maintain fixation and do not miss any of the central color changes, even a single missed probe on the c-VFT indicates that caution is warranted when using visual test materials.

\section{Acknowledgments}

This work was supported by the Independent Research Fund Denmark under the Sapere Aude program (Grant ID: DFF - 4180-00201 to R.S), and the Fakutsi support group. We thank Alex Leff for enabling patient recruitment and commenting on the program features during the pilot phase.

Medical technical assistant Dorthe Thulstrup Nyegaard offered valuable help with the Humphrey Visual Field Analyzer. 


\section{References}

Ali, M., Hazelton, C., Lyden, P., Pollock, A., \& Brady, M. (2013). Recovery from poststroke visual impairment: evidence from a clinical trials resource. Neurorehabilitation and Neural Repair, 27(2), 133-141.

Carl Zeiss Meditec Inc. (2003). Humphrey Field Analyzer II - i series: User's Guide. Dublin, CA: Carls Zeiss Meditec Inc.

Dzwiniel, P., Gola, M., Wójcik-Gryciuk, A., \& Waleszczyk, W. J. (2017).

Specvis: Free and open-source software for visual field examination. PLoS One, 12(10), e0186224.

Esterman, B. (1982). Functional scoring of the binocular field. Ophthalmology, 89(11), 1226-1234.

Hepworth, L., \& Rowe, F. J. (2016). Visual impairment following stroke-the impact on quality of life: A systematic review. Ophthalmology Research: An International Journal, 5(2), 1-15.

Koiava, N., Ong, Y. H., Brown, M. M., Acheson, J., Plant, G. T., \& Leff, A. P. (2012). A ‘web app' for diagnosing hemianopia. Journal of Neurology, Neurosurgery \& Psychiatry, 83(12), 1222-1224.

Koo, T. K., \& Li, M. Y. (2016). A guideline of selecting and reporting intraclass correlation coefficients for reliability research. Journal of Chiropractic Medicine, 15(2), $155-163$

Neumann, G., Schaadt, A. K., Reinhart, S., \& Kerkhoff, G. (2016). Clinical and psychometric evaluations of the cerebral vision screening questionnaire in 461 nonaphasic individuals poststroke. Neurorehabilitation and Neural Repair, 30(3), 187198. 
Olsen, A. S., Alberti, M., Serup, L., la Cour, M., Damato, B., \& Kolko, M.

(2016). Glaucoma detection with damato multifixation campimetry online. Eye, 30(5),

731-739.

Pandit, R. J., Gales, K., \& Griffiths, P. G. (2001). Effectiveness of testing visual fields by confrontation. The Lancet, 358(9290), 1339-1340.

Peirce, J. W. (2007). PsychoPy - Psychophysics software in Python. Journal of Neuroscience Methods, 162(1-2), 8-13

Peirce, J. W. (2009). Generating stimuli for neuroscience using PsychoPy. Frontiers in Neuroinformatics, 2(10), doi: 10.3389/neuro.11.010.2008.

Rowe, F., Brand, D., Jackson, C. A., Price, A., Walker, L., Harrison, S., ... \& Howard, C. (2009). Visual impairment following stroke: do stroke patients require vision assessment? Age and Ageing, 38(2), 188-193.

Shrout, P. E., \& Fleiss, J. L. (1979). Intraclass correlations: uses in assessing rater reliability. Psychological Bulletin, 86(2), 420-428.

Zihl, J. (2011). Rehabilitation of Visual Disorders after Brain Injury: 2nd Edition. Neuropsychological Rehabilitation: A Modular Handbook. East Sussex, UK: Psychology Press. 